\title{
LRG1 in pancreatic cancer cells promotes inflammatory factor synthesis and the angiogenesis of HUVECs by activating VEGFR signaling
}

\author{
Duxiong Cai ${ }^{1}$, Chunji Chen ${ }^{2}$, Yexiong Su${ }^{1}$, Yan Tan ${ }^{1}$, Xuyue Lin ${ }^{1}$, Rong Xing ${ }^{1}$ \\ ${ }^{1}$ Department of Gastroenterology, The First Affiliated Hospital of Hainan Medical University, Haikou, China; ${ }^{2}$ Department of Pathology, Hainan \\ Provincial People's Hospital, Haikou, China \\ Contributions: (I) Conception and design: D Cai, C Chen; (II) Administrative support: D Cai; (III) Provision of study materials or patients: D Cai, C \\ Chen, Y Su; (IV) Collection and assembly of data: D Cai, C Chen, Y Su, Y Tan, X Lin; (V) Data analysis and interpretation: D Cai, C Chen, Y Tan, \\ X Lin, R Xing; (VI) Manuscript writing: All authors; (VII) Final approval of manuscript: All authors. \\ Correspondence to: Duxiong Cai, PhD. Department of Gastroenterology, The First Affiliated Hospital of Hainan Medical University, 31 Longhua Rd, \\ Longhua District, Haikou 570102, China. Email: caiduxiongcdx@163.com.
}

Background: This study aimed to investigate the roles of leucine-rich alpha-2-glycoprotein 1 (LRG1) in regulating angiogenesis during pancreatic cancer $(\mathrm{PC})$ pathogenesis.

Methods: LRG1 expression in tissues was detected by qRT-PCR and immunohistochemistry. LRG1 in BxPC-3 and Capan-2 cells was knocked down or overexpressed. Cell viability and the migration and invasion abilities of cells were analyzed using the Cell Counting Kit-8 (CCK-8) assay and Transwell system, respectively. Interleukin-1 beta (IL-1 $\beta$ ), IL-18, and vascular endothelial growth factor A (VEGFA) contents in cell culture were measured by ELISA, and the angiogenesis of HUVECs was assessed by the in vitro tube formation assay. In vitro LRG1 expression in BxPC-3 and Capan-2 cells was determined using immunofluorescence.

Results: The results showed that LRG1 expression was significantly increased in pancreatic cancer tissues and cell lines. LRG1 knockdown inhibited the viability, migration, invasion, and IL-1 $\beta$ and IL-18 synthesis of BxPC-3 and Capan-2 cells. VEGFA synthesis in BxPC-3 and Capan-2 cells was also inhibited by LRG1 knockdown, which caused impaired tube formation of co-cultured HUVECs. LRG1 overexpression enhanced the viability, migration, and invasion of BxPC-3 and Capan-2 cells, also causing elevated tube formation of HUVECs and IL-1 $\beta$ and IL-18 synthesis in co-cultures of HUVECs and BxPC-3 or Capan-2 cells. Silencing of VEGF receptor (VEGFR) abrogated the enhanced tube formation and IL-1 $\beta$ and IL-18 synthesis in HUVECs co-cultured with BxPC-3 or Capan-2 cells overexpressing LRG1.

Conclusions: In conclusion, LRG1, which is highly expressed in pancreatic cancer cells, promotes inflammatory factor synthesis and the angiogenesis of HUVECs though activating the VEGFR signaling pathway.

Keywords: Leucine-rich alpha-2-glycoprotein 1 (LRG1); pancreatic cancer; angiogenesis; inflammation; vascular endothelial growth factor receptor (VEGFR)

Submitted Nov 30, 2021. Accepted for publication Jan 30, 2022.

doi: 10.21037/jgo-21-910

View this article at: https://dx.doi.org/10.21037/jgo-21-910 


\section{Introduction}

Pancreatic cancer is one of the most common human malignancies. It is characterized by rapid progression and a high mortality rate, and is listed as one of the leading causes of cancer-related death worldwide $(1,2)$. A recent epidemiological analysis demonstrated that pancreatic cancer was estimated to cause more than 44,000 and 79,000 deaths annually in the United States and China, respectively, and its incidence is still increasing especially in developed countries $(3,4)$. Pancreatic ductal adenocarcinoma (PDAC) is the major subtype of pancreatic cancer. It accounts for over $95 \%$ of all global pancreatic cancer cases and has a 5 -year survival rate of less than 5\% (2,5). More importantly, pancreatic cancer is notoriously difficult to treat, mainly due to its typically late diagnosis at the metastatic stage, lack of an effective cure, and high relapse rates following surgical resection $(1,2,5)$. Extensive investigations have shown that the pathogenesis of pancreatic cancer is predominantly driven by genetic mutations in major signaling components, such as Kirsten rat sarcoma viral oncogene homolog (KRAS), cyclin-dependent kinase inhibitor $2 \mathrm{~A}$ gene $(\mathrm{CDKN} 2 \mathrm{~A})$, which is alternatively called p16, tumor suppressor protein-53 (TP53), and mothers against decapentaplegic homolog $4(\operatorname{Smad} 4)(2,6)$. However, the molecular events driving pancreatic cancer initiation and progression, especially metastasis which contributes to its high malignancy, still remain poorly understood.

Angiogenesis refers to the formation of new blood vessels. It plays essential roles in various physiological processes and pathological conditions such as embryonic and fetal development, bone regeneration, and would healing, as well as regenerative medicine and tissue engineering $(7,8)$. Angiogenesis has been regarded as a risk factor in pancreatic cancer (9). Previous reports have established that the processes of angiogenesis are modulated by a panel of chemical stimuli and cell factors, such as vascular endothelial growth factor (VEGF), plateletderived growth factor (PDGF), fibroblast growth factor (FGF), hypoxia-inducible factor (HIF), and transforming growth factor beta (TGF- $\beta$ ) $(7,8,10)$. Specifically, VEGF is known to perform its angiogenic functions by activating the tyrosine kinase-mediated signaling networks through directly binding with 3 VEGF receptors (VEGFRs), which are widely expressed in vascular smooth muscle cells, vascular endothelial cells, and other cell types involved in angiogenesis $(8,10,11)$. In the context of cancer progression, VEGFA levels in tumor tissues are usually elevated to a maximum degree at the vascular sprout sites. VEGFA binds with VEGFR2 to enhance signaling events, promoting cell proliferation and vascular permeability, which finally contribute to angiogenesis and cancer progression and metastasis $(8,9,12)$. For instance, the inhibition of VEGFR expression and activation was shown to effectively suppress tube formation and lymphatic endothelial cell sprouting, along with angiogenesis and pancreatic cancer growth and progression (13). Meanwhile, angiogenesis in human cancers may also be initiated and modulated by hypoxia and inflammation $(10,14)$. Therefore, elucidation of the molecular mechanisms underlying inflammation and angiogenesis in pancreatic cancer may facilitate the development of new therapeutic strategies.

Leucine-rich alpha-2-glycoprotein 1 (LRG1) is a serum glycoprotein consisting of 312 amino acids with leucinerich repeats. It has been shown to regulate cell adhesion, apoptosis, and migration, and is associated with various biological processes such as would healing, ischemia/ reperfusion injury, osteoarthritis, and cancer initiation and progression (15-19). More importantly, LRG1 has been shown to be a key regulator of inflammation and angiogenesis under distinct contexts (18,20-23). For instance, the angiogenesis and migration of mesenchymal stem cells in subchondral bones during osteoarthritis were shown to be significantly promoted by tumor necrosis factor-alpha (TNF- $\alpha$ )-induced LRG1 expression (18). Also, LRG1-regulated angiogenesis processes could be effectively induced by TGF- $\beta$, which has been implicated in various pathogenic conditions including ischemia and diabetic kidney disease progression (20-22). In addition, recent reports also revealed that LRG1 could enhance angiogenesis to regulate cancer growth and migration $(23,24)$. Specifically, the angiogenesis and epithelialmesenchymal transition processes in colorectal cancer were shown to be promoted by LRG1 through activation of the expression of hypoxia-inducible factor 1 alpha $($ HIF-1 $\alpha)(23)$. However, little is known about the potential roles of LRG1 in angiogenesis processes during pancreatic cancer pathogenesis.

In the present study, we aimed to investigate the expression and roles of LRG1 in pancreatic cancer cells, as well as its influence on the tube formation ability of human vascular epithelial cells. In this study, we firstly found that LRG1 can promoted pancreatic cancer through HUVEC. This study may provide new insights into the molecular mechanisms regulating angiogenesis processes associated with pancreatic cancer development and progression. 
Table 1 Primers used for qRT-PCR analysis

\begin{tabular}{llr}
\hline Gene & Primer sequences $\left(5^{\prime}-3^{\prime}\right)$ & Product length (bp) \\
\hline LRG1 forward & TGTTCGTCATGGGTGTGAAC & 154 \\
LRG1 reverse & ATGGCATGGACTGTGGTCAT & 170 \\
VEGFR forward & TTACTTGCAGGGGACAGAGG & 226 \\
VEGFR reverse & TTCCCGGTAGAAGCACTTGT & \\
$\beta$-actin forward & GGACACCCTGGTATTGAAAGAAA & \\
$\beta$-actin reverse & TAGCCGTTCTAATTGCAGCGG & \\
\hline
\end{tabular}

We present the following article in accordance with the MDAR checklist (available at https://jgo.amegroups.com/ article/view/10.21037/jgo-21-910/rc).

\section{Methods}

\section{Patient cobort and bioinformatics}

Serum samples were collected from 15 patients diagnosed with PDAC treated at the Department of Gastroenterology, The First Affiliated Hospital of Hainan Medical University between July 2018 and May 2019. Serum samples collected from another 15 healthy volunteers were used as the control group. The correlation between LRG1 expression and survival in pancreatic cancer was analyzed using the online Kaplan-Meier plotter (KMplot) database. Relative LRG1 expression between cancerous and adjacent noncancerous tissues in pancreatic cancer patients was analyzed by searching the GEPIA (Gene Expression Profiling Interactive Analysis) database. All procedures performed in this study involving human participants were in accordance with the Declaration of Helsinki (as revised in 2013). The study was approved by the ethics committee of the First Affiliated Hospital of Hainan Medical University (No. 2000-227) and informed consent was taken from all the patients.

\section{Cell culture and co-culture}

Five pancreatic cancer cell lines BxPC-3, Panc-1, CFPAC, Capan-2, and AsPC-1, the human normal pancreatic duct epithelial cell line HPDE6-C7, and HUVECs (human umbilical vein endothelial cells) were purchased from the Shanghai Cell Bank, Chinese Academy of Science (Shanghai, China). Cells were cultured in DMEM (Dulbecco's Modified Eagle's medium; Thermo Fisher Scientific, Waltham,
MA, USA) supplemented with 5\% [fetal bovine serum (FBS); Thermo Fisher Scientific] at $37^{\circ} \mathrm{C}$ in a humidified incubator with a supply of $5 \% \mathrm{CO}_{2}$. To analyze the impact of pancreatic cancer cells on the tube formation and expression of cytokines, we used a model cell for blood vessel formation, HUVECs in this study. Pancreatic cancer cells and HUVECs were cultured in a Transwell chamber system.

\section{Quantitative RT-PCR (qRT-PCR)}

Relative mRNA expression levels in patient serum and cultured cells were detected by the qRT-PCR method using total RNA samples extracted using the RNAprep Pure Cell/ Bacteria Kit (\#DP430; Tiangen Biotech, Beijing, China) according to the manufacturer's protocol. The cDNA library for the quantitative PCR assay was then synthesized from $2.5 \mu \mathrm{g}$ RNA samples of each group using the FastQuant RT Super Mix (\#KR108; Tiangen Biotech, Beijing, China) as per the manufacturer's instructions. The expressional levels of mRNAs were then measured by real-time quantitative PCR using the Talent Fluorescent Quantitation Detection Kit-SYBR Green (\#FP209; Tiangen Biotech, Beijing, China) following the manufacturer's protocol. Relative mRNA expression levels were finally calculated via the standard $2^{-\Delta \Delta \mathrm{Ct}}$ method following normalized the expression of $\beta$-actin. Information of primers used for qRT-PCR analysis are shown in Table 1.

\section{Cell transfection}

For knockdown of LRG1 and VEGFR expression in pancreatic cancer cells or HUVECs, the siRNA sequences LRG1 siRNA\#1 (5'-GCUGCAAUUGAACGGCUA dTdT-3'), LRG1 siRNA\#2 (5'-CCUGGAUCUGUGACCAGAA dTdT$\left.3^{\prime}\right)$, and the corresponding negative control (NC) sequences (5'-GUGCACUAUCUAGAGGACA dTdT-3'), and VEGFR 
siRNA (5'-GAUGAAAGUUACCAGUCUA dTdT-3') and the NC sequences (5'-UGCAGAAUCCGAAUACGCU dTdT3'), were all synthesized by Sangon Biotech Company (Shanghai, China). For overexpression of LRG1, the coding sequences (CDS) of LRG1 were ligated with the pcDNA3.0 plasmid for establishing the recombinant pcDNA3.0-LRG1 vector. These siRNA sequences and vectors were transfected into cultured pancreatic cancer cells or HUVECs with the Lipofectamine 3000 reagent (\#L3000001; Thermo Fisher Scientific) as per the manufacturer's protocol.

\section{Cell viability}

The viability of BxPC-3 and Capan-2 cells after transfection and treatment was assessed in this study using the Cell Counting Kit (CCK-8/WST-8) (\#AMJ-KT0001; AmyJet Scientific, Wuhan, China) following the manufacturer's instructions. Briefly, approximately 3,000 cells $(100 \mu \mathrm{L})$ from each group were seeded in 96-well plates and cultured at $37{ }^{\circ} \mathrm{C}$ for $24 \mathrm{~h}$. Subsequently, $100 \mu \mathrm{L}$ CCK-8 reagent was added to each well followed by incubation at $37{ }^{\circ} \mathrm{C}$ for another $2 \mathrm{~h}$. The viability of pancreatic cancer cells was finally assessed by measuring the OD450 (absorbance at $450 \mathrm{~nm}$ ) using a microplate reader.

\section{Cell migration and invasion}

The migration and invasion abilities of cultured BxPC3 and Capan-2 cells were detected in this study using the Transwell culture system (Corning). Briefly, BxPC-3 and Capan-2 cells were placed into the upper chamber filled with DMEM medium containing no FBS at $37^{\circ} \mathrm{C}$ for 48 h. Cells which migrated into the lower chamber filled with DMEM supplemented with $10 \%$ FBS were then fixed with $4 \%$ paraformaldehyde for $15 \mathrm{~min}$ and stained with $0.5 \%$ crystal violet for $10 \mathrm{~min}$, followed by imaging and quantification of cell migration under a microscope. The invasion ability of cultured BxPC-3 and Capan-2 cells was determined following similar procedures except that the inner surfaces of the Transwell upper chamber were precoated with Matrigel matrix (Corning).

\section{ELISA}

The relative contents of LRG1, IL-1 $\beta$, IL-18, and VEGFA in serum samples and cell supernatants were quantified by ELISA (enzyme-linked immunosorbent assay) using the Human LRG1 ELISA Kit (\#E-EL-H1287c), Human interleukin-1 beta (IL-1 $\beta$ ) ELISA Kit (\#E-EL-H0149c), Human IL-18 ELISA Kit (\#E-EL-H0253c), and Human VEGFA ELISA Kit (\#E-EL-H0111c), respectively, following the experimental procedures provided by the manufacturer. Their contents in serum and cell culture supernatants were biologically repeated for at least three biological replicates.

\section{Immunofuorescence}

The in vitro expression of VEGFA in BxPC-3 and Capan-2 cells was detected by immunofluorescence staining. Briefly, cultured BxPC-3 and Capan-2 cell were fixed and permeabilized with $4 \%$ paraformaldehyde for $12 \mathrm{~min}$ at room temperature and blocked with $5 \%$ BSA (bovine serum albumin) for $40 \mathrm{~min}$ at room temperature. Cells were then incubated with anti-VEGFA antibody (\#ab52917; Abcam; 1:500) for $1 \mathrm{~h}$ at room temperature and FITCconjugated anti-rabbit IgG antibody for another $1 \mathrm{~h}$ at room temperature. The expression of VEGFA in pancreatic cancer cells in vitro was finally determined by observation under a laser confocal microscope.

\section{Tube formation assay}

The angiogenesis potential of HUVECs was evaluated by the in vitro tube formation assay as previously described (25). Briefly, 96-well plates were pre-coated with Matrigel matrix (50 $\mu \mathrm{L}$ matrix each well) followed by polymerization at $37{ }^{\circ} \mathrm{C}$ for $50 \mathrm{~min}$. Approximately $3 \times 10^{4}$ cultured HUVECs in normal medium were seeded in each well after specified treatments, followed by incubation at $37^{\circ} \mathrm{C}$ under normal conditions for another 6-8 h. The tubular structures formed by the HUVECs in 96-well plates were finally observed under microscopy.

\section{Hematoxylin and eosin staining}

The tissues collected from PC patients were immersed in $4 \%$ paraformaldehyde at $4{ }^{\circ} \mathrm{C}$ overnight. Immersionfixed tissues were embedded in paraffin and sectioned at $5 \mu \mathrm{m}$. Subsequently, slides were dehydrated and dewaxed by ethanol and xylene, respectively, followed by staining with hematoxylin and eosin $(\mathrm{H} \& \mathrm{E})$. Finally, the stained slides were sealed with neutral resin and observed and recorded under a light microscope.

\section{Immunobistocbemistry}

Paraffin sections were dehydrated and dewaxed by ethanol 
and xylene, respectively. Tissues were blocked using $5 \%$ BSA for $30 \mathrm{~min}$ at room temperature. The primary antibody (LRG1, ab178698, 1:200) and secondary antibody (DAKO, K5007) were added one after another and covered the tissue on the slide. Then, slides were color developed by DAB. Finally, hematoxylin was used to stain the cell nucleus before sealing with neutral resin. Slides were observed and imaged under a light microscope.

\section{Statistical analysis}

Quantitative data from at least 3 biological replicates, presented as mean \pm standard deviation, were analyzed by SPSS 20.0 software for statistical significance. Differences between 2 or more groups were evaluated by the Student's $t$-test or ANOVA method. Significant differences were defined by $\mathrm{P}<0.05$.

\section{Results}

Elevated LRG1 expression was associated with poor pancreatic cancer survival

To explore the involvement of LRG1 in angiogenesis and pancreatic cancer progression, we first analyzed the expression of LRG1 in cancerous tissues from pancreatic cancer patients and cell lines, as well as its correlation with the survival of pancreatic cancer patients. Through searching the KMplot database, we found that the survival of pancreatic cancer patients with high LRG1 expression was significantly lower than those with low LRG1 expression (Figure 1A). Also, expression data from the GEPIA database showed that the expression levels of LRG1 in cancerous tissues of pancreatic cancer patients were markedly higher than the corresponding adjacent noncancerous pancreatic tissues (Figure 1B). Pancreatic cancer tissue and adjacent non-cancerous tissue was stained using the H\&E method as shown in Figure 1C. The expression of LRG1 in pancreatic cancer tissue and adjacent noncancerous tissue was detected using immunohistochemistry (Figure 1D). Using ELISA, we found that the LRG1 expression levels in the serum samples of 15 pancreatic cancer patients were markedly higher than those of healthy volunteers (Figure 1E). Consistent with this, we also found that the expression of LRG1 in 5 pancreatic cancer cell lines, BxPC-3, Panc-1, CFPAC, Capan-2 and AsPC-1, was significantly increased compared with the human normal pancreatic duct epithelial cell line HPDE6-C7 (Figure 1F).
Among the cell lines, the highest expression of LRG1 was observed in BxPC-3 and Capan-2 cells, which were then used for the subsequent assays. The highly elevated LRG1 expression in clinical tissues and cell lines suggests the potential roles of LRG1 in regulating pancreatic cancer progression and angiogenesis.

\section{LRG1 knockdown suppressed pancreatic cancer cell viability, migration, invasion, and the secretion of inflammatory factors}

To analyze the influence of LRG1 on pancreatic cell functions, we knocked down the expression of LRG1 in 2 pancreatic cancer cell lines by transfection with specific siRNAs (Figure 2A). We found that silencing LRG1 expression significantly repressed the viabilities of both BxPC-3 and Capan-2 cells compared with the blank and NC groups (Figure 2B,2C). Moreover, the Transwell assay showed that the migration abilities of both BxPC-3 and Capan-2 cells were significantly impaired by the transfection of siRNAs targeting LRG1 expression (Figure 2D). Similarly, the invasion abilities of BxPC-3 and Capan-2 cells after LRG1 knockdown were significantly repressed compared to the blank and NC group (Figure 2E). In addition, the secretion of 2 important inflammatory factors, IL-1 $\beta$ and IL-18, by both BxPC-3 and Capan-2 cells was greatly inhibited by LRG1 knockdown (Figure 2F,2G). Further results of western blot assay showed LRG1 knockdown by siRNA suppressed expression of $\mathrm{N}$-cadherin and Vimentin, but promoted E-cadherin (Figure 2H). These results suggest that LRG1 can promote pancreatic cancer proliferation, migration, invasion, and the synthesis of inflammatory factors.

\section{LRG1 knockdown suppressed VEGFA expression in pancreatic cancer cells and the tube formation of HUVECs}

To further investigate the effects of LRG1 expression on angiogenesis associated with cancer progression, we firstly obtained the expression of VEGFR in pancreatic cancer based on GEPIA database. Result showed that VEGFR was relative higher in tumor tissues (Figure $3 A$ ). Then results detected the expression of VEGFA in pancreatic cancer cells with LRG1 knockdown and the alteration of tube formation of HUVECs co-cultured pancreatic cancer cells. Result of qRT-PCR showed that expression of VEGFR was relative higher in $\mathrm{BxPC} 3$, but lower in Capan-2 cell line (Figure 3B). While, VEGFR was also elevated in PC tissues 
A

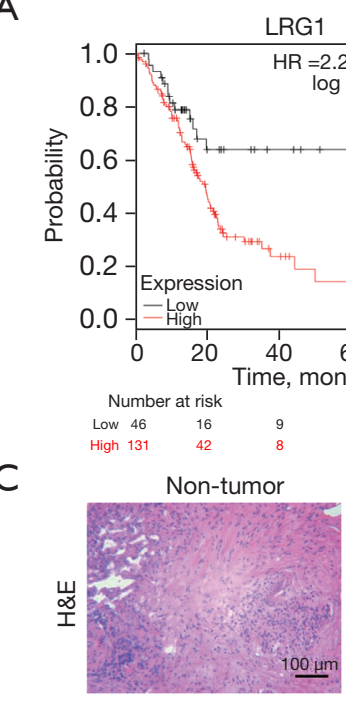

$\mathrm{E}$

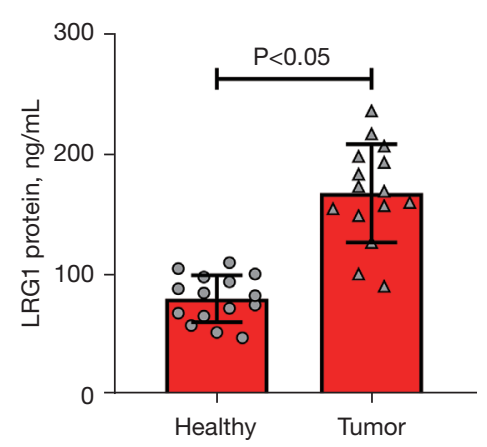

B

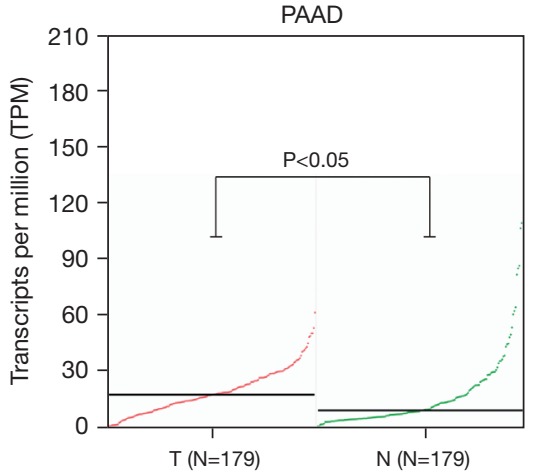

D Non-tumor Tumor

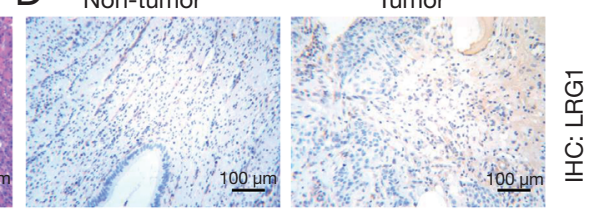

$\mathrm{F}$

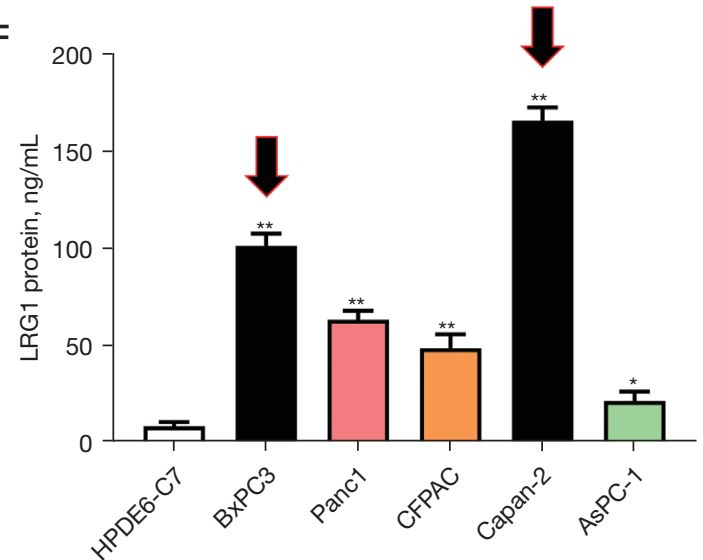

Figure 1 Increased LRG1 expression in pancreatic cancer tissues and cell lines. (A) The correlation between LRG1 expression and the survival of pancreatic cancer patients. Data from the KMplot database was used to generate survival curves. (B) The differential expression of LRG1 in cancerous and adjacent non-cancerous tissues from pancreatic cancer patients. The expression of LRG1 in pancreatic cancer patients was analyzed using the GEPIA database. (C) H\&E staining was used to investigate the pathology of pancreatic cancer tissues and adjacent non-cancerous tissues. (D) LRG1 expression was detected using immunohistochemistry in pancreatic cancer tissues and adjacent non-cancerous tissues. (E) LRG1 content was increased in the serum of pancreatic cancer patients. LRG1 levels in serum samples from 15 pancreatic cancer patients and another 15 healthy volunteers were detected by the ELISA method. (F) Significant elevation of LRG1 expression in pancreatic cancer cell lines. The mRNA expression levels of LRG1 in BxPC-3, Panc-1, CFPAC, Capan-2, AsPC-1, and HPDE6-C7 cells were detected by the quantitative RT-PCR method. *, $\mathrm{P}<0.05$; **, $\mathrm{P}<0.01$, compared with HPDE6-C7. LRG1, leucinerich alpha-2-glycoprotein 1; KMplot, Kaplan-Meier plotter; GEPIA; Gene Expression Profiling Interactive Analysis; H\&E, hematoxylin and eosin.

compared to para-carcinoma tissue (Figure S1). We found that the in vitro expression levels of VEGFA in both BxPC3 and Capan-2 cells were significantly lower compared with the blank and NC groups (Figure 3C). Immunofluorescence detection showed that LRG1 siRNA suppressed expression of VEGFA in BxPC3 and Capan-2 cells (Figure 3D,3E).
Also, the tube formation of HUVECs co-cultured with both BxPC-3 and Capan-2 cells was greatly suppressed by the silencing of LRG1 expression in these 2 pancreatic cancer cell lines (Figure 3F). Furthermore, using ELISA, we demonstrated that the secretion of VEGFA by the coculture of HUVECs with BxPC-3 or Capan-2 cells was 

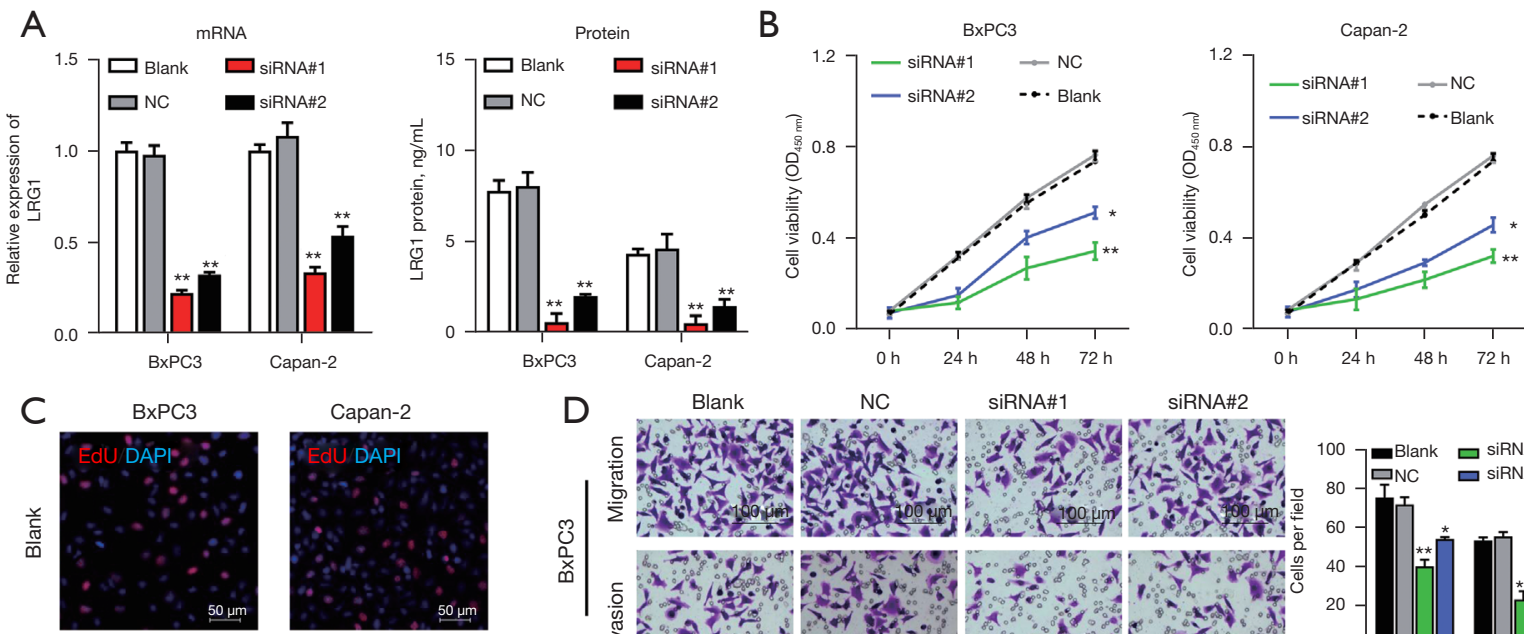

NC

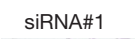

SiRNA\#2
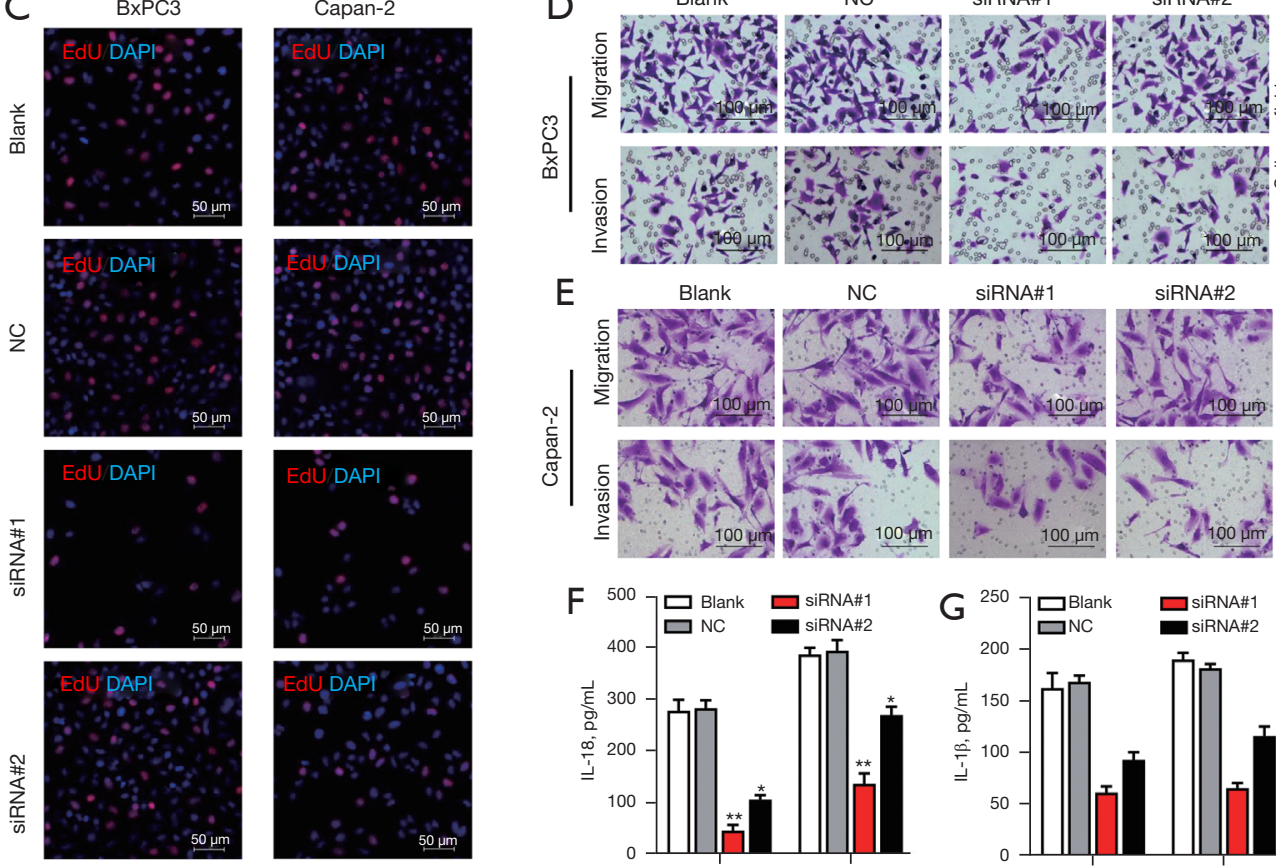

SiRNA\#1

SiRNA\#2
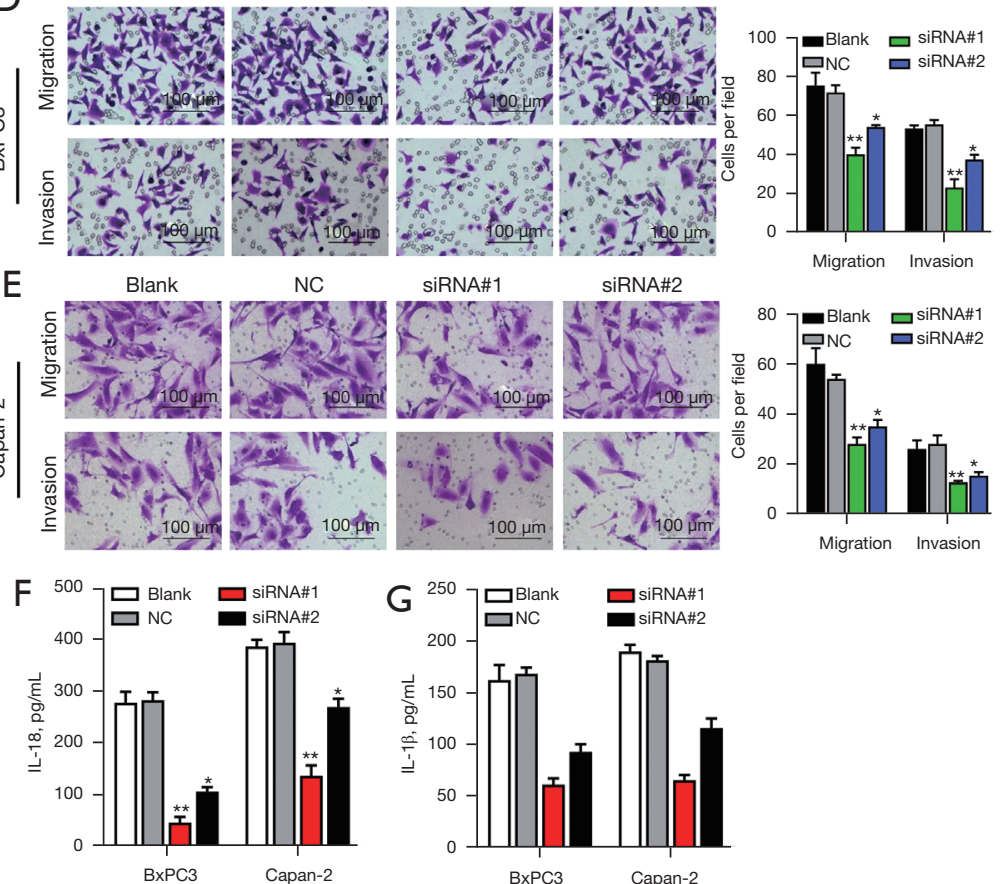

$\mathrm{H}$
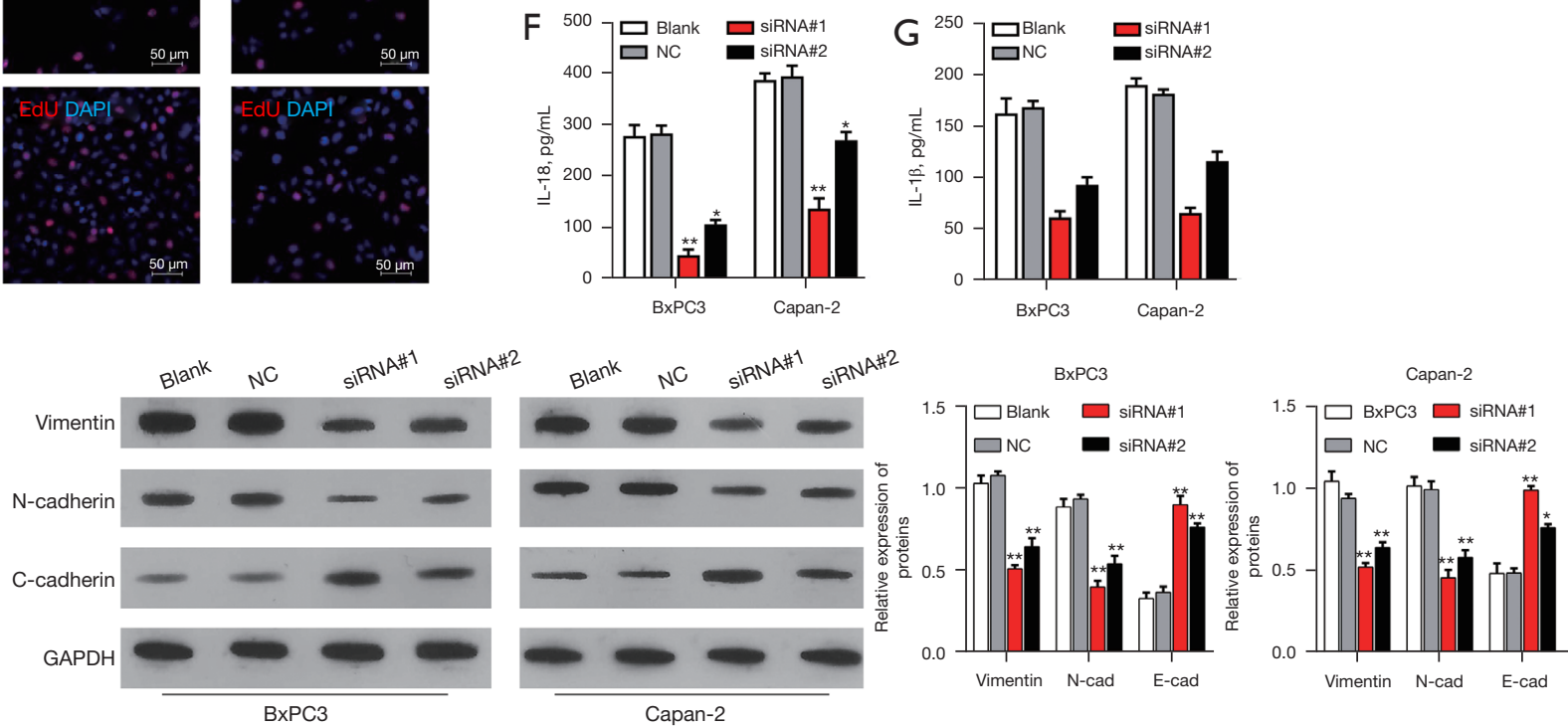

Figure 2 Inhibition of pancreatic cancer cell viability, migration, invasion, and inflammatory factor secretion by LRG1 knockdown. (A) Relative expression of LRG1 in BxPC-3 and Capan-2 cells transfected with siRNAs targeting LRG1. The mRNA expression levels in cells were assessed by the quantitative RT-PCR method. (B,C) The effects of LRG1 knockdown on the viabilities of BxPC-3 and Capan-2 cells by using CCK8 assay (B) and EdU staining (C). (D,E) Suppression of BxPC-3 and Capan-2 cell migration and invasion by siRNAs targeting LRG1 expression. The migration (D) and invasion (E) abilities of cancer cells were detected using the Transwell assay. Cells were stained with crystal violet. (F,G) Inhibition of the secretion of inflammatory factors in BxPC-3 and Capan-2 cells by the silencing of LRG1 expression. The contents of IL-1 $\beta$ and IL-18 in cell culture were determined by the ELISA method. (H) Expression of Vimentin, N-cadherin and E-Cadherin was measured by using Western blot assay. *, $\mathrm{P}<0.05$; ${ }^{* *}, \mathrm{P}<0.01$, compared with NC. LRG1, leucine-rich alpha-2glycoprotein 1 ; NC, negative control. 
effectively inhibited by the knockdown of LRG1 expression in pancreatic cancer cells (Figure 3G). These results showed that LRG1 expression in pancreatic cancer cells could promote VEGFA synthesis and promote angiogenesis during pancreatic cancer progression.

\section{LRG1 overexpression enbanced pancreatic cancer cell migration and invasion and promoted inflammatory factor synthesis in HUVECs via VEGFR}

To validate the pathogenic roles of LRG1 in angiogenesis during pancreatic cancer development, we subsequently overexpressed LRG1 in BxPC-3 and Capan-2 cells (Figure 4). LRG1 overexpression in BxPC-3 and Capan-2 cells was verified by ELISA assay (Figure $4 A$ ). Through the CCK-8 assay, we observed that the viabilities of both BxPC3 and Capan-2 cells were significantly enhanced by LRG1 overexpression compared with the NC group (Figure 4B, $4 C$ ). Moreover, the migration rates of BxPC-3 and Capan-2 cells were also significantly elevated by LRG1 overexpression compared with the NC group. Similar enhancement of the invasion abilities of both BxPC-3 and Capan-2 cells was caused by overexpression of LRG1 in these 2 pancreatic cancer cell lines (Figure 4D,4E). To investigate whether LRG1-induced VEGFA secretion by pancreatic cancer cells could regulate inflammatory factor synthesis in HUVECs, we silenced the expression of VEGFR in HUVECs, which were then co-cultured with pancreatic cancer cells overexpressing LRG1. We showed that synthesis of IL$1 \beta$ and IL-18 by HUVECs was significantly enhanced by co-culture with BxPC-3 or Capan-2 cells overexpressing LRG1, which was then effectively abrogated by the silencing of VEGFR in HUVECs (Figure 4F,4G). Finally, the expression of Vimentin, N-cadherin and E-cadherin was measured by Western blot assay, and results showed that LRG1 overexpression induced promotion of N-cadherin and Vimentin. While E-cadherin was inhibited by LRG1 overexpression (Figure 4H). These results indicated that LRG1-induced VEGF secretion in pancreatic cancer cells could effectively promote the synthesis of inflammatory factors in HUVECs via VEGFR.

\section{LRG1 overexpression in pancreatic cancer cells promoted the tube formation of HUVECs through activating VEGFR}

Finally, we analyzed the alteration of tube formation of HUVECs induced by LRG1 overexpression in pancreatic cancer cells. We showed that the tube formation of
HUVECs could be significantly enhanced by co-culture with the pancreatic cancer cell lines BxPC-3 and Capan-2 (Figure 5A). Moreover, the tube formation of HUVECs was enhanced to an even greater level by co-culture with BxPC-3 and Capan-2 cells with overexpression of LRG1, compared with those co-cultured with pancreatic cancer cells without LRG1 overexpression (Figure 5A). However, the enhanced tube formation of HUVECs caused by coculture with LRG1-overexpressing BxPC-3 and Capan-2 cells was significantly abrogated by the silencing of VEGFR expression in HUVECs with specific siRNAs (Figure 5A). In addition, we showed that the synthesis of VEGFA in the co-culture of HUVECs and BxPC-3 or Capan-2 cells was also greatly increased by LRG1 overexpression in these 2 pancreatic cancer cells (Figure 5B). Together, these results showed that LRG1 expression could enhance VEGFA synthesis in pancreatic cancer cells, which promoted angiogenesis during pancreatic cancer progression by activating VEGFR in vascular epithelial cells.

\section{Discussion}

The current clinical management of pancreatic cancer, especially the major subtype PDAC, is mainly dependent on surgical resection combined with chemotherapy, radiotherapy, and targeted therapy $(1,2)$. The global survival rate of pancreatic cancer patients has not been effectively improved over the past decades, mainly because of its high malignancy which manifests as rapid metastasis and high relapse rates $(1,2)$. As introduced above, angiogenesis in tumor tissues and associated inflammation play essential roles in the progression and metastasis of pancreatic cancer $(10,13,14)$. However, the molecular events driving inflammation and angiogenesis during pancreatic cancer progression remain poorly understood. In the present study, we showed that the expression of LRG1, a serum glycoprotein associated with angiogenesis under distinct contexts, was highly expressed in pancreatic cancer tissues and cell lines. Moreover, the expression of LRG1 could positively regulate the viability, migration, invasion, and VEGFA synthesis of pancreatic cancer cells, shown by the silencing and overexpression of LRG1 in 2 pancreatic cancer cell lines. Importantly, we demonstrated that coculture with pancreatic cancer cells effectively promoted the secretion of inflammatory factors and tube formation of HUVECs, which was mediated by the activation of VEGFR in HUVECs. These results have established LRG1 as a critical factor driving angiogenesis during pancreatic cancer 
A

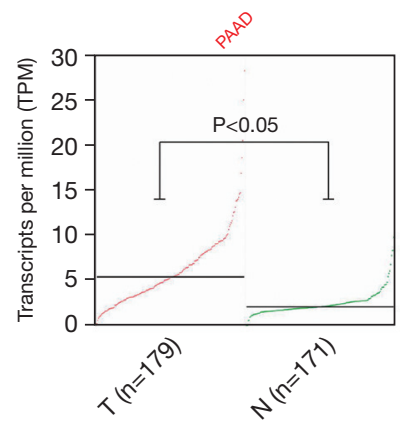

Blank

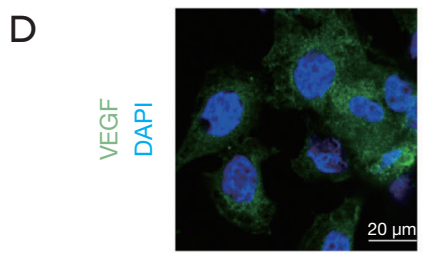

E

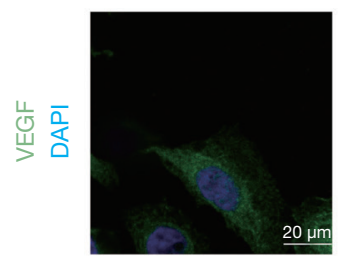

F

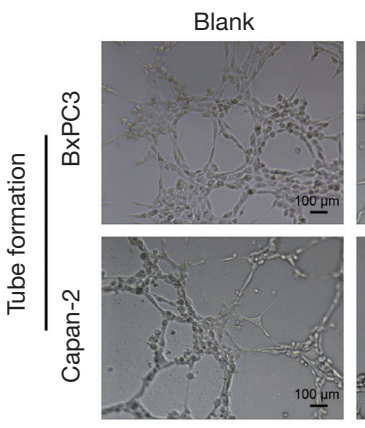

B

NC

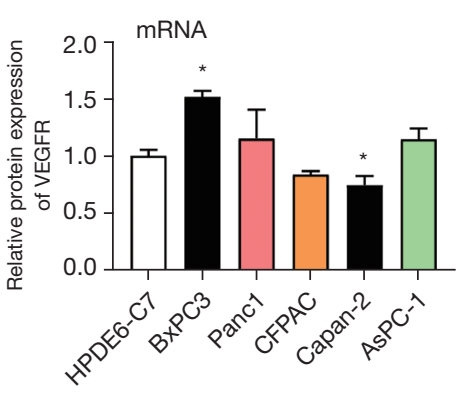

NC
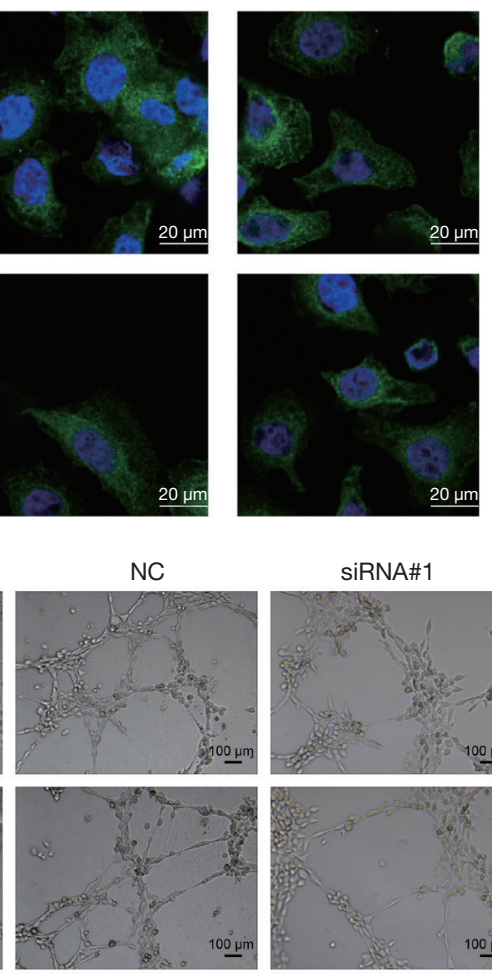

SiRNA\#1

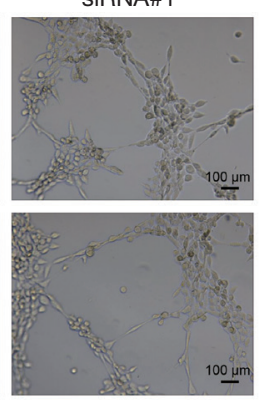

SiRNA\#1
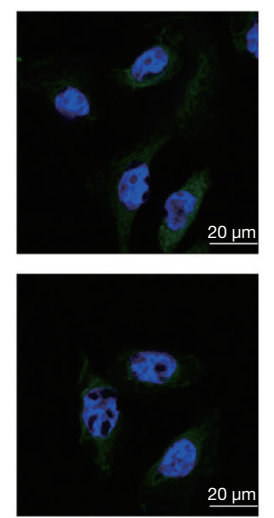

SiRNA\#2
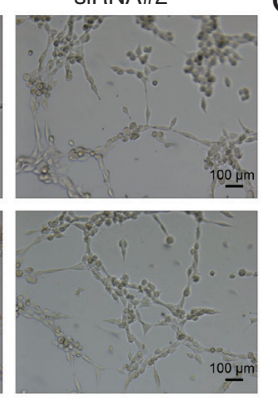
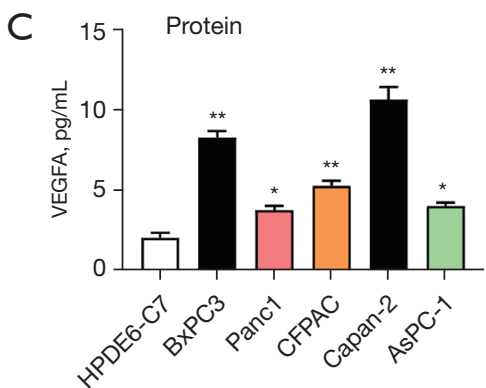

SiRNA\#2
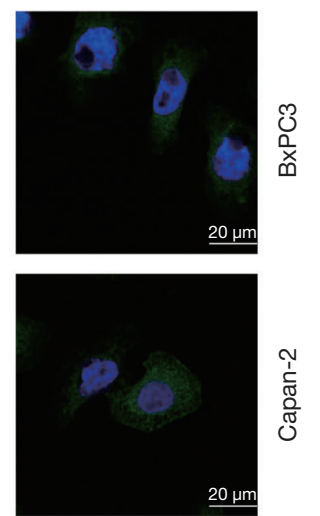

G

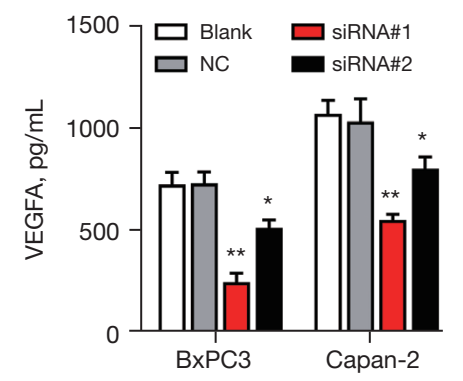

Figure 3 Suppressed VEGFA synthesis and tube formation of HUVECs co-cultured with pancreatic cancer cells with LRG1 knockdown. (A) Expression of VEGFR in pancreatic cancer based on GEPIA database. (B) Expression of VEGFR was measured in cell lines by using qRT-PCR. (C) The in vitro expression levels of VEGFA in both BxPC-3 and Capan-2 cells with knockdown of LRG1 expression. VEGFA expression in pancreatic cancer cells was measured by immunofluorescence. (D,E) VEGFA expression in BxPC3 and Capan-2 was detected after LRG1 knockdown. (F) The effects of LRG1 silencing in BxPC-3 and Capan-2 cells on the tube formation of co-cultured HUVECs. HUVECs were used as an in vitro angiogenesis model. (G) The secretion of VEGFA by the co-cultured HUVECs and pancreatic cancer cells with knockdown of LRG1 expression. The content of VEGFA in cell cultures was quantified by the ELISA method. *, P<0.05; **, $\mathrm{P}<0.01$, compared with NC. LRG1, leucine-rich alpha-2-glycoprotein 1; VEGFA, vascular endothelial growth factor A; NC, negative control; HUVECs, human umbilical vein endothelial cells.

progression.

The elevated expression of LRG1 associated with cancer progression has been extensively reported in previous studies, including ovarian cancer $(15,26)$, gastric cancer (24), esophageal squamous cell carcinoma (17), colorectal cancer (23), glioma (19), and endometrial carcinoma (27). Also, the altered expression of LRG1 has been suggested as a biomarker for predicting the prognosis of cancer patients 

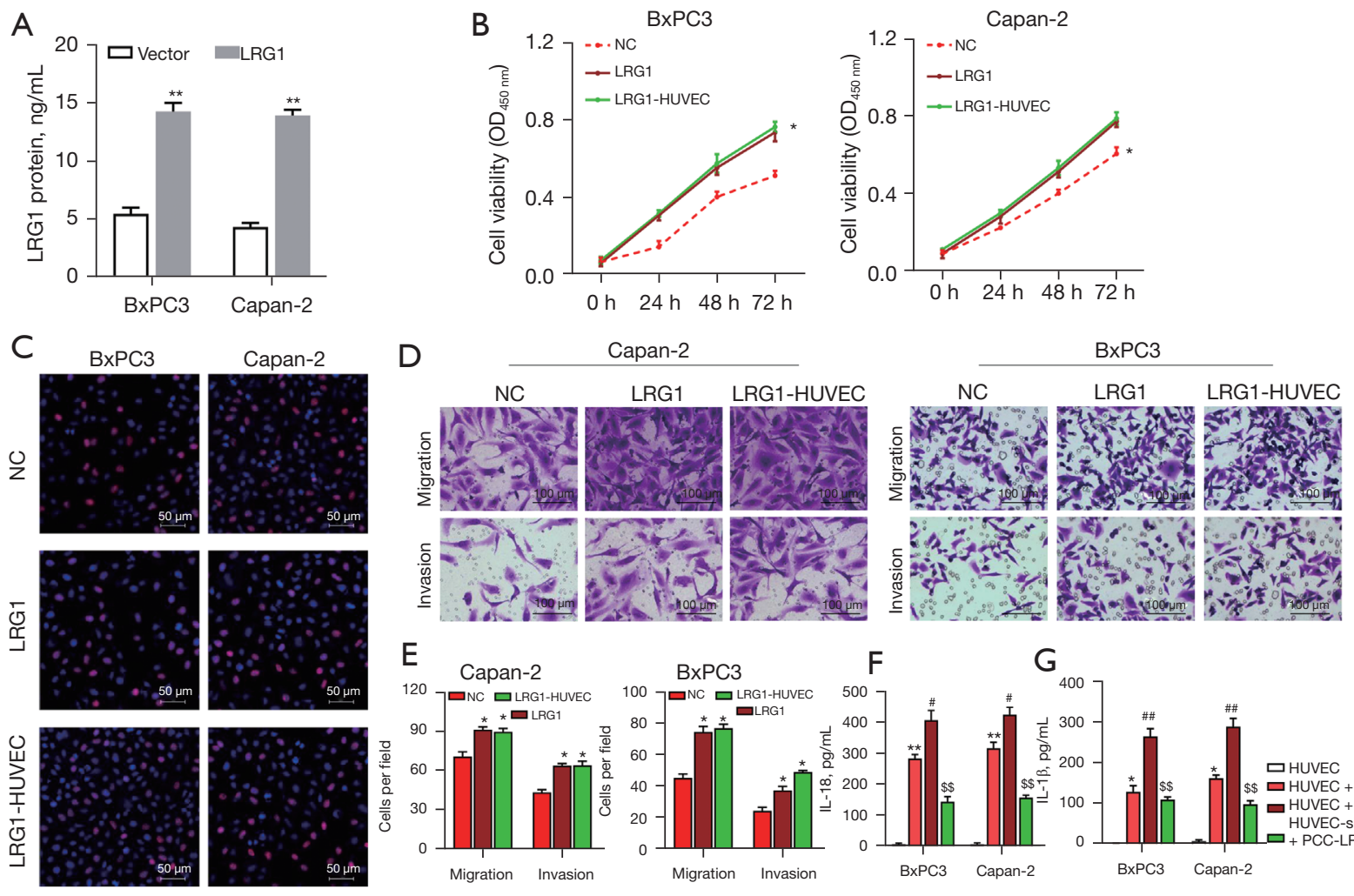

G

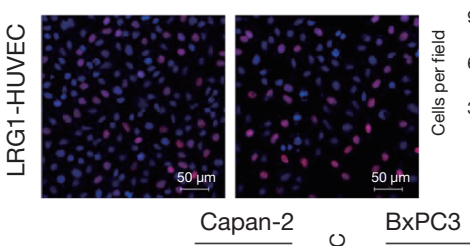

㟧
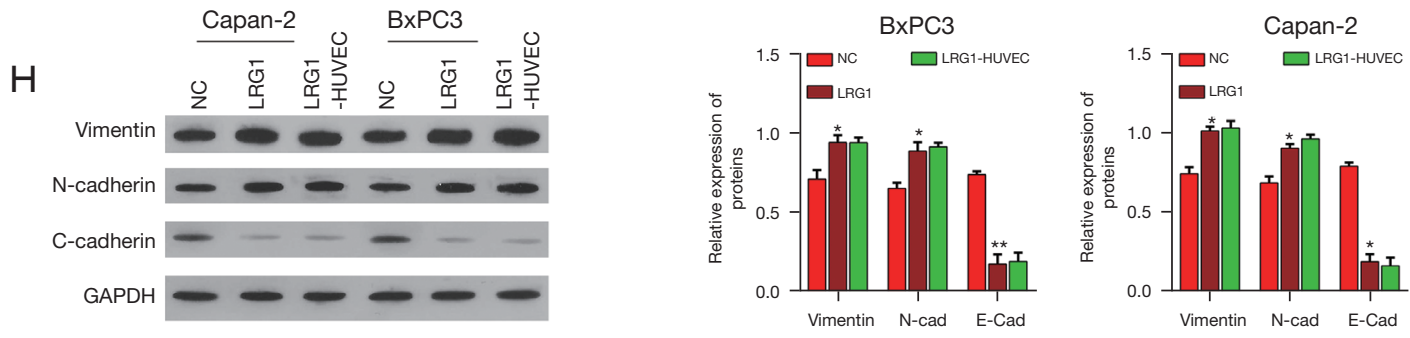

Figure 4 Enhanced pancreatic cancer cell migration and invasion and inflammatory factor synthesis of HUVECs by LRG1 overexpression. (A) Overexpression of LRG1 was verified by ELISA assay. (B,C). The viabilities of pancreatic cancer cells were altered by overexpression of LRG1. BxPC-3 (A) and Capan-2 cell viabilities were measured by the CCK-8 assay (B) and EdU staining $(\mathrm{C})$. * $\mathrm{P}<0.05,{ }^{* *}, \mathrm{P}<0.01$ compared with NC. (D,E) Enhanced migration and invasion abilities of pancreatic cancer cell lines by LRG1 overexpression. The migration rates and invasion ability of BxPC-3 and Capan-2 cells were evaluated using the Transwell system. Cells were stained with crystal violet. *, $\mathrm{P}<0.05$, compared with NC. (F,G) The influence of VEGFR silencing in HUVECs on inflammatory factor synthesis by HUVECs cocultured with pancreatic cancer cells overexpressing LRG1. IL-1 $\beta$ (F) and IL-18 (G) contents in the cell co-culture were measured by the ELISA method. *, $\mathrm{P}<0.05$, ${ }^{* *}, \mathrm{P}<0.01$, compared with HUVECs; ${ }^{*}, \mathrm{P}<0.05$, ${ }^{\# \#}, \mathrm{P}<0.01$, compared with HUVECs + PCC-Vector; ${ }^{\text {\$ }}$, $\mathrm{P}<0.01$, compared with HUVECs + PCC-LRG1. (H) Expression of Vimentin, N-cadherin and E-cadherin was measured by Western bolt assay. LRG1, leucine-rich alpha-2-glycoprotein 1; NC, negative control; HUVECs, human umbilical vein endothelial cells; VEGFR, vascular endothelial growth factor receptor.

(24,26,27). However, little is known about its expression changes and pathogenic roles in pancreatic cancer initiation and progression, as well as its potential as a biomarker for pancreatic cancer patients. In the present study, based on bioinformatics analysis and our own experiments, we showed that the expression of LRG1 in pancreatic cancer tissues and cell lines was significantly elevated, and was correlated with the poor survival of pancreatic cancer patients. This greatly increased expression indicates the potential of LRG1 as a new candidate biomarker for 

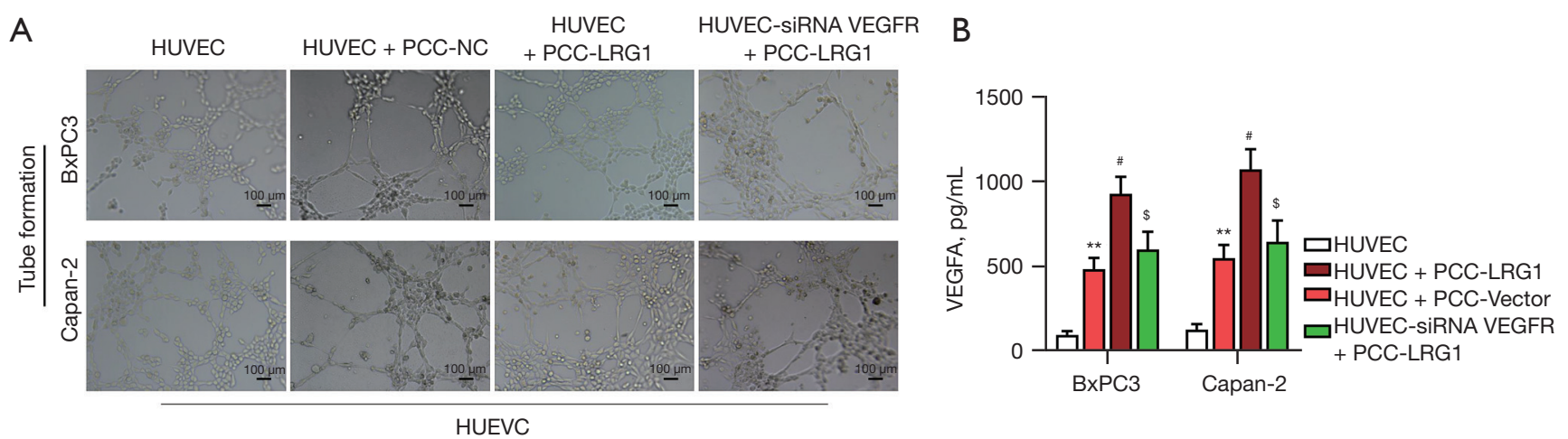

Figure 5 Enhanced tube formation of HUVECs by LRG1 overexpression in pancreatic cancer cells was mediated by VEGFR in HUVECs. (A) The altered tube formation of HUVECs with knockdown of VEGFR following co-culture with LRG1-overexpressing pancreatic cancer cells. The tube formation assay was used as an in vitro angiogenesis model for evaluating the ability of pancreatic cancer cells to regulate angiogenesis during tumorigenesis. (B) The VEGFA contents in the co-culture of LRG1-overexpressing BxPC-3 or Capan-2 cells and HUVECs with knockdown of VEGFR expression. **, $\mathrm{P}<0.01$, compared with HUVECs; *, $\mathrm{P}<0.05$, compared with HUVECs + PCCVector; $\$$, P<0.05, compared with HUVECs + PCC-LRG1. LRG1, leucine-rich alpha-2-glycoprotein 1; HUVECs, human umbilical vein endothelial cells; NC, negative control; VEGFA, vascular endothelial growth factor A; VEGFR, vascular endothelial growth factor receptor.

pancreatic cancer diagnosis and prognostic prediction, which requires further evaluation by larger-scale clinical studies. Our subsequent analysis also confirmed that overexpression of LRG1 could promote pancreatic cancer cell viability, migration, and invasion, while silencing LRG1 resulted in completely opposite effects on pancreatic cancer cell functions. These in vitro assays indicated the critical roles of high LRG1 expression in pancreatic initiation and progression, suggesting that LRG1 may be a promising new target for pancreatic cancer diagnosis and treatment, which deserves further verification using in vivo animal cancer models.

Although LRG1 was originally discovered to be a regulator of various cellular processes such as proliferation, apoptosis, migration, and invasion $(16,17,19)$, it has been characterized as key modulator of angiogenesis associated with cancer pathogenesis in recent years $(18,20,23)$. However, the regulation of angiogenesis by LRG1 expression during pancreatic cancer development has not been addressed in previous reports. In the present study, we used HUVECs as an in vitro angiogenesis model. HUVECs were used for co-culture experiments with pancreatic cancer cells with altered LRG1 expression. We clearly showed that co-culture with pancreatic cancer cells could enhance the tube formation of HUVECs, which was greatly suppressed by LRG1 silencing and promoted by LRG1 overexpression in pancreatic cancer cells. These investigations demonstrate the pathogenic functions of LRG1 in driving angiogenesis associated with pancreatic cancer progression. From previous reports, it is known that the regulation of angiogenesis by LRG1 is mediated by the TGF-b signaling pathway (20-22). Meanwhile, the pathogenic process of pancreatic cancer has also been shown to be mediated by significant tumorigenic alterations of the TGF-b signaling networks (28-30). These findings indicate that angiogenesis processes in the context of pancreatic cancer could also possibly be regulated by the TGF-b signaling pathway, which warrants further investigations.

As introduced above, angiogenesis is linked to cancer metastasis and progression and is usually triggered by VEGFs (1). It is also associated with inflammation and altered synthesis of inflammatory factors $(10,14)$, and inflammatory factors play a role in exacerbating the development of pancreatic cancer (31). Researchers have been demonstrated that IL-18 and IL- $1 \beta$ promotes pancreatic cancer progression and metastasis. Sun et al recently discover that Pin 1 activates NF- $\mathrm{NB}$ pathway and promotes IL-18 production by interacting with $\mathrm{P} 65$ in pancreatic cancer (32). besides, research supports that IL-1 $\beta$ promotes pancreatic ductal adenocarcinoma through a immune suppression (33). In the present study, we showed that the expression of VEGFA as well as the synthesis of 2 inflammatory factors, IL-1 $\beta$ and IL-18, in pancreatic cancer cells could be greatly repressed by the knockdown of LRG1 expression, indicating the roles of LRG1 in promoting VEGFA secretion and inflammatory factor production in 
pancreatic cancer cells. Also, the IL-1 $\beta$ and IL-18 levels in the co-culture of LRG1-overexpressing pancreatic cancer cells and HUVECs could be significantly reduced by the silencing of VEGFR expression in HUVECs, showing that VEGF produced by pancreatic cancer cells in response to LRG1 overexpression also stimulates inflammatory factor production in HUVECs. Furthermore, the enhanced tube formation of HUVECs induced by co-culture with LRG1overexpressing pancreatic cancer cells was shown to be effectively abrogated by silencing VEGFR expression in HUVECs. This observation demonstrates that the angiogenesis process enhanced by LRG1 expression in pancreatic cancer cells is mediated by the binding of VEGF with VEGFR in HUVECs and the activation of signaling pathways downstream of VEGFR in HUVECs. Combined with the already known roles of VEGR in pancreatic cancer $(13,34,35)$, our discovery further highlights the applicability of VEGFR inhibition as a preventive strategy for pancreatic cancer progression.

In summary, we revealed that LRG1, which was highly expressed in pancreatic cancer tissues and cell lines, promoted the migration, invasion, and production of VEGF and inflammatory factors in pancreatic cancer cells, and could enhance the tube formation of HUVECs by activating VEGFR signaling. These results provide new insights into the molecular mechanisms of angiogenesis processes underlying pancreatic cancer progression and metastasis, and may provide targets for the development of new therapeutic drugs for advanced cancer patients.

\section{Acknowledgments}

Funding: This research was supported by Hainan Provincial Natural Science Foundation of China (818MS145).

\section{Footnote}

Reporting Checklist: The authors have completed the MDAR checklist. Available at https://jgo.amegroups.com/article/ view/10.21037/jgo-21-910/rc

Data Sharing Statement: Available at https://jgo.amegroups. com/article/view/10.21037/jgo-21-910/dss

Conflicts of Interest: All authors have completed the ICMJE uniform disclosure form (available at https://jgo.amegroups. com/article/view/10.21037/jgo-21-910/coif). The authors have no conflicts of interest to declare.
Ethical Statement: The authors are accountable for all aspects of the work in ensuring that questions related to the accuracy or integrity of any part of the work are appropriately investigated and resolved. All procedures performed in this study involving human participants were in accordance with the Declaration of Helsinki (as revised in 2013). The study was approved by the ethics committee of the First Affiliated Hospital of Hainan Medical University (No. 2000-227) and informed consent was taken from all the patients.

Open Access Statement: This is an Open Access article distributed in accordance with the Creative Commons Attribution-NonCommercial-NoDerivs 4.0 International License (CC BY-NC-ND 4.0), which permits the noncommercial replication and distribution of the article with the strict proviso that no changes or edits are made and the original work is properly cited (including links to both the formal publication through the relevant DOI and the license). See: https://creativecommons.org/licenses/by-nc-nd/4.0/.

\section{References}

1. Grant TJ, Hua K, Singh A. Molecular Pathogenesis of Pancreatic Cancer. Prog Mol Biol Transl Sci 2016;144:241-75.

2. Tanaka S. Molecular Pathogenesis and Targeted Therapy of Pancreatic Cancer. Ann Surg Oncol 2016;23 Suppl 2:S197-205.

3. Chen W, Zheng R, Baade PD, et al. Cancer statistics in China, 2015. CA Cancer J Clin 2016;66:115-32.

4. Siegel RL, Miller KD, Jemal A. Cancer statistics, 2018. CA Cancer J Clin 2018;68:7-30.

5. Khan MA, Azim S, Zubair H, et al. Molecular Drivers of Pancreatic Cancer Pathogenesis: Looking Inward to Move Forward. Int J Mol Sci 2017;18:779.

6. Mann KM, Ying H, Juan J, et al. KRAS-related proteins in pancreatic cancer. Pharmacol Ther 2016;168:29-42.

7. Ambrose CT. Pro-Angiogenesis Therapy and Aging: A Mini-Review. Gerontology 2017;63:393-400.

8. Teleanu RI, Chircov C, Grumezescu AM, et al. Tumor Angiogenesis and Anti-Angiogenic Strategies for Cancer Treatment. J Clin Med 2019;9:84.

9. Li S, Xu HX, Wu CT, et al. Angiogenesis in pancreatic cancer: current research status and clinical implications. Angiogenesis 2019;22:15-36.

10. Aguilar-Cazares D, Chavez-Dominguez R, Carlos-Reyes A, et al. Contribution of Angiogenesis to Inflammation and Cancer. Front Oncol 2019;9:1399. 
11. Liu K, Ren T, Huang Y, et al. Apatinib promotes autophagy and apoptosis through VEGFR2/STAT3/BCL2 signaling in osteosarcoma. Cell Death Dis 2017;8:e3015.

12. Zhang Z, Ji S, Zhang B, et al. Role of angiogenesis in pancreatic cancer biology and therapy. Biomed Pharmacother 2018;108:1135-40.

13. Chen HM, Tsai CH, Hung WC. Foretinib inhibits angiogenesis, lymphangiogenesis and tumor growth of pancreatic cancer in vivo by decreasing VEGFR-2/3 and TIE-2 signaling. Oncotarget 2015;6:14940-52.

14. Palma G, Barbieri A, Bimonte S, et al. Interleukin 18: friend or foe in cancer. Biochim Biophys Acta 2013;1836:296-303.

15. Andersen JD, Boylan KL, Jemmerson R, et al. Leucinerich alpha-2-glycoprotein-1 is upregulated in sera and tumors of ovarian cancer patients. J Ovarian Res 2010;3:21.

16. Gao Y, Xie Z, Ho C, et al. LRG1 Promotes Keratinocyte Migration and Wound Repair through Regulation of HIF$1 \alpha$ Stability. J Invest Dermatol 2020;140:455-464.e8.

17. Wang Y, Xing Q, Chen X, et al. The Clinical Prognostic Value of LRG1 in Esophageal Squamous Cell Carcinoma. Curr Cancer Drug Targets 2019;19:756-63.

18. Wang Y, Xu J, Zhang X, et al. TNF- $\alpha$-induced LRG1 promotes angiogenesis and mesenchymal stem cell migration in the subchondral bone during osteoarthritis. Cell Death Dis 2017;8:e2715.

19. Zhong D, He G, Zhao S, et al. LRG1 modulates invasion and migration of glioma cell lines through TGF- $\beta$ signaling pathway. Acta Histochem 2015;117:551-8.

20. Hong Q, Zhang L, Fu J, et al. LRG1 Promotes Diabetic Kidney Disease Progression by Enhancing TGF- $\beta$ Induced Angiogenesis. J Am Soc Nephrol 2019;30:546-62.

21. Meng H, Song Y, Zhu J, et al. LRG1 promotes angiogenesis through upregulating the TGF- $\beta 1$ pathway in ischemic rat brain. Mol Med Rep 2016;14:5535-43.

22. Wang X, Abraham S, McKenzie JAG, et al. LRG1 promotes angiogenesis by modulating endothelial TGF- $\beta$ signalling. Nature 2013;499:306-11.

23. Zhang J, Zhu L, Fang J, et al. LRG1 modulates epithelialmesenchymal transition and angiogenesis in colorectal cancer via HIF-1 $\alpha$ activation. J Exp Clin Cancer Res 2016;35:29.

24. Yamamoto M, Takahashi T, Serada S, et al. Overexpression of leucine-rich $\alpha 2$-glycoprotein-1 is a prognostic marker and enhances tumor migration in gastric cancer. Cancer Sci 2017;108:2052-60.

25. Huang JJ, Shi YQ, Li RL, et al. Angiogenesis effect of therapeutic ultrasound on HUVECs through activation of the PI3K-Akt-eNOS signal pathway. Am J Transl Res 2015;7:1106-15.

26. Wu J, Yin H, Zhu J, et al. Validation of LRG1 as a potential biomarker for detection of epithelial ovarian cancer by a blinded study. PLoS One 2015;10:e0121112.

27. Wen SY, Zhang LN, Yang XM, et al. LRG1 is an independent prognostic factor for endometrial carcinoma. Tumour Biol 2014;35:7125-33.

28. Javle M, Li Y, Tan D, et al. Biomarkers of TGF- $\beta$ signaling pathway and prognosis of pancreatic cancer. PLoS One 2014;9:e85942.

29. Conejo-Garcia JR. Breaking barriers for T cells by targeting the EPHA2/TGF- $\beta / \mathrm{COX}-2$ axis in pancreatic cancer. J Clin Invest 2019;129:3521-3.

30. Otsuru T, Kobayashi S, Wada H, et al. Epithelialmesenchymal transition via transforming growth factor beta in pancreatic cancer is potentiated by the inflammatory glycoprotein leucine-rich alpha-2 glycoprotein. Cancer Sci 2019;110:985-96.

31. Padoan A, Plebani M, Basso D. Inflammation and Pancreatic Cancer: Focus on Metabolism, Cytokines, and Immunity. Int J Mol Sci 2019;20:676.

32. Sun Q, Fan G, Zhuo Q, et al. Pin 1 promotes pancreatic cancer progression and metastasis by activation of NF- $\kappa \mathrm{B}-$ IL-18 feedback loop. Cell Prolif 2020;53:e12816.

33. Takahashi R, Macchini M, Sunagawa M, et al. Interleukin$1 \beta$-induced pancreatitis promotes pancreatic ductal adenocarcinoma via B lymphocyte-mediated immune suppression. Gut 2021;70:330-41.

34. Kurenova E, Liao J, He DH, et al. The FAK scaffold inhibitor C4 disrupts FAK-VEGFR-3 signaling and inhibits pancreatic cancer growth. Oncotarget 2013;4:1632-46.

35. Peng $\mathrm{X}$, Wang $\mathrm{Y}, \mathrm{Li} \mathrm{H}$, et al. ATG5-mediated autophagy suppresses NF- $\mathrm{B}$ signaling to limit epithelial inflammatory response to kidney injury. Cell Death Dis 2019;10:253.

(English Language Editor: C. Betlazar-Maseh)

Cite this article as: Cai D, Chen C, Su Y, Tan Y, Lin X, Xing R. LRG1 in pancreatic cancer cells promotes inflammatory factor synthesis and the angiogenesis of HUVECs by activating VEGFR signaling. J Gastrointest Oncol 2022;13(1):400-412. doi: 10.21037/jgo-21-910 

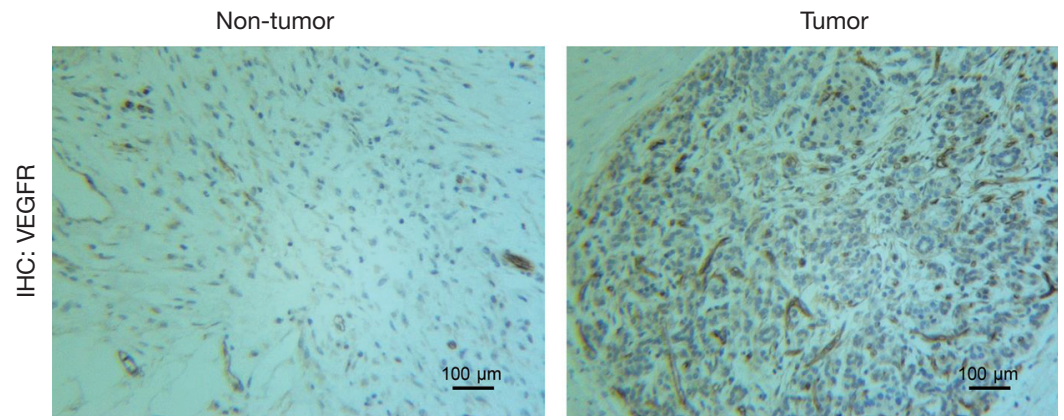

Figure S1 Expression of VEGFR was detected in tumor tissues and paracancer tissues by using immunohistochemistry (magnification, 100x). Tissues were stained by using DAB developing kit. 